\title{
The ASDEX Upgrade Program Targeting Gaps to Fusion Energy
}

\author{
R. Neu*, V. Bobkov, A. Bock, M. Bernert, M. \\ Beurskens, A. Herrmann, A. Kallenbach, P.T. Lang, \\ J.-M. Noterdaeme\#, G. Pautasso, M. Reich, \\ J. Schweinzer, J. Stober, W. Suttrop, H. Zohm \\ Max-Planck-Institut für Plasmaphysik, \\ Boltzmannstr.2, 85748 Garching, Germany \\ *Technische Universität München, \\ Boltzmannstr.15, 85748 Garching, Germany \\ \# Ghent University, Ghent, Belgium
}

\author{
A. Kirk \\ CCFE, Culham Science Centre, \\ Abingdon, OX14 3DB, United Kingdom \\ ASDEX Upgrade Team \\ and EUROfusion MST1 Team§
}

\begin{abstract}
Recent experiments in ASDEX Upgrade aimed at improving the physics base for ITER and DEMO to prepare operation and aid the design. In order to increase its exhaust capabilities and operational flexibility a new bulk $W$ divertor as well as an adjustable cryo-pump had been installed prior to the 2014 campaign. In experiments with high field side pellet injection central electron densities twice as high as the Greenwald density limit could be achieved without strongly increasing the pedestal density and deleterious effect on confinement. Due to its large installed heating power a large normalized heat flux $P_{\text {sep }} / R=10 \mathrm{MWm}^{-1}$ has been reached, representing $2 / 3$ of the ITER value, under partially detached conditions with a peak target heat flux well below $10 \mathrm{MWm}^{-2}$. The divertor load could be further reduced by increasing the core radiation still keeping the confinement in the range of $\mathrm{H}_{98 \mathrm{y} 2} \approx 1$. Suppression of edge localized modes (ELMs) at low collisionality has been observed in a narrow spectral window in contrast to earlier results at high densities. The ITER $Q=10$ baseline scenario has been investigated, matching as close as possible the triangularity, the plasma beta, $q_{95}$ and the distance the L-H threshold. It turned out that the ELMs frequency is low and consequently the energy ejected by a single ELM is very high and ELM mitigation appears to be difficult. As a possible alternative a scenario has been developed achieving a similar performance at lower plasma current (and consequently higher $\mathbf{q}_{95}$ ). Experiments using ECCD with feedback controlled deposition have allowed successfully testing several control strategies for ITER, including automated control of $(3,2)$ and $(2,1)$ NTMs during a single discharge. Concerning advanced scenarios, experiments with central ctr-ECCD have been per-formed in order to modify the q-profile. A strong reversal of the q-profile could be stationary achieved and an internal transport barrier could be triggered. In disruption mitigation studies with massive gas injection (MGI) a runaway electron beam could be provoked and mitigated by a second MGI. Ongoing enhance-ments aim at the strengthening of the power supplies in order to allow full use of the installed heating power, the exchange of two ICRH antennas to reduce the $W$ influx during ICRH and the upgrading of the ECRH system to 7-8 MW for 10s.
\end{abstract}

Keywords - tokamak; power exhaust; plasma scenarios; disruptions

$\S$ see http://www.euro-fusionscipub.org/mst1

\section{INTRODUCTION}

Since 2014 the all-tungsten ASDEX Upgrade (AUG) tokamak $(\mathrm{R}=1.65 \mathrm{~m}, \mathrm{a}=0.5, \mathrm{\kappa}=1.6)$ is run as a European device under the newly established EUROfusion consortium, devoting about half of its operational time to experiments guided by the 'Medium-Sized Tokamak' task-force [1]. One of the guidelines for the recent experiments is the European Fusion Roadmap [2] aimed at improving the physics base for ITER and DEMO to aid the design and prepare operation. The roadmap identifies power exhaust as a major issue, specifically in DEMO. This is true not only for the steady state power exhaust which will increase by a factor 5-6 compared to ITER, but also for the expected large ELMs which have the potential to destroy the plasma facing components. Another issue to be solved for a DEMO reactor is the steady state operation which will rely on efficient ways for current drive and scenario tailoring.

In order to increase its exhaust capabilities and operational flexibility a new bulk $\mathrm{W}$ divertor as well as an adjustable cryopump had been installed prior to the 2014 campaign [3]. In the following sections a selection of the investigations in support of ITER and DEMO using the strengths and the dedicated tools of AUG is presented. ITER and DEMO shall operate close to or just above the Greenwald density $\left(\mathrm{n}_{\mathrm{GW}}\right)$. Even in present day devices it is difficult to reach this density in H-Mode discharges with gas puff only. In ITER and DEMO pellet fuelling will be mandatory in order to deposit the fuel inside the separatrix. Sec. IIA will present the latest results of the high field side (HFS) pellet injection experiments at ASDEX Upgrade. According to the latest results from scaling experiments [4], the power decay length in ITER and DEMO will be very similar to that in AUG. As a consequence, the power density in the divertor will scale with the machine size as the power crossing the separatrix divided by the length of the circumference $\left(\mathrm{P}_{\mathrm{sep}} / \mathrm{R}\right)$. Due to its huge installed heating power $\left(\mathrm{P}_{\text {aux }}=34 \mathrm{MW}\right)$ a large normalized heat flux $\mathrm{P}_{\text {sep }} / \mathrm{R}$ can be achieved in AUG. Sec. IIB will describe the latest experiments where a record value of $\mathrm{P}_{\text {sep }} / \mathrm{R}$ has been reached still keeping the power load in the divertor acceptable by radiative cooling. Sec. III presents the investigations on the ITER baseline scenario, matching $\mathrm{n} / \mathrm{n}_{\mathrm{GW}}, \beta, \delta, \mathrm{q}_{95}$ and the 
distance to the L-H threshold, but consequently not matching $\nu^{*}$ and $\rho^{*}$. A longstanding problem is the periodical power load by edge localized modes (ELMs) which is expected to be deleterious to the divertor plasma facing components in ITER. Amongst the several methods for ELM mitigation, magnetic perturbation (MP) coils are thought to be an important tool, although the mechanism for the mitigation or suppression of ELMs is not yet completely clarified. Sec. IV reports on the latest achievements in AUG at low collisionality. Since more than a decade it is well known that neoclassical tearing modes (NTMs) could degrade the confinement and that dedicated external current drive could be a remedy. Sec. VA shows the successful application of an automated tool for tracking the mode position and positioning the electron cyclotron current drive (ECCD). In order to operate a DEMO in steady state, means for external current drive as well as a large fraction of boot strap current will be necessary. Since the timescale for current diffusion in AUG is rather short and the off-axis current drive capabilities are limited because of the lower temperatures compared to DEMO, on axis counter-ECCD is explored in AUG in order to tailor the current profile (see Sec. VB). Finally, Sec. VI will describe the latest experiments on the suppression of runaway electrons (RE) in specifically designed discharges where a RE beam is provoked by a first massive gas injection (MGI) and mitigated by a second one. In Sec. VII an outlook on the upcoming hardware upgrades and in Sec. VIII a summary and corresponding conclusions are given.

\section{HIGH DENSITY OPERATION AND POWER EXHAUST AT HIGHEST P/R VALUES}

\section{A. Operation Above the Greenwald Density by High-Field Side Pellet Injection}

Operation in a future fusion reactor will aim to establish a high core density in order to reach a maximum output power. In addition, a high separatrix density is essential for power exhaust. Therefore, in the ITER project and in the European DEMO studies, core fueling is envisaged entirely by injecting pellets formed from frozen fuel from the torus inboard side. The separatrix density will most likely be adjustable by gas puff in these devices, leading to a certain degree of freedom in the control scheme of core and separatrix/divertor density. AUG, equipped with an efficient inboard pellet fueling system, a sophisticated system for flexible real time plasma control and a versatile set of diagnostics, is very suited for such investigations.

In AUG a simultaneous real-time control of the edge and the core density has been achieved by using the neutral density in the divertor $\left(\mathrm{n}_{0}{ }^{\text {div }}\right)$ and a combination of Bremsstrahlung measurements and a central $\mathrm{CO}_{2}$ interferometer chord. By employing these control parameters, core densities at about 2 times $\mathrm{n}_{\mathrm{Gw}}$ have been established by pellet injection while the separatrix density was kept stable by controlled reduction of the initial gas puff rate [5]. In discharge \#29674 ( $\mathrm{I}_{\mathrm{P}}=1.0 \mathrm{MA}$, $\mathrm{q}_{95}=4.6, \mathrm{P}_{\mathrm{NBI}}=7.4 \mathrm{MW}, \mathrm{P}_{\mathrm{ICRH}}=2.5 \mathrm{MW}$ ) pellets were launched with $570 \mathrm{~m} / \mathrm{s}$ at $70 \mathrm{~Hz}$ resulting in a particle flux at the launcher exit of $2.6 \cdot 10^{22} \mathrm{D} / \mathrm{s}$. No significant loss of confinement takes place during the pellet induced high density phase; afterwards the discharge reversibly returns to its initial conditions. Due to the soft termination of this discharge, a full recovery of the pellet perturbed data from the DCN laser interferometer for all chords was achieved for the post pellet phase making a reconstruction of the evolution of the density profile possible. The ratio of innermost channel (H-1) to edge channel (H-5, see Fig.1) shows usual post pellet decay of density profile peaking. However, the ratio of $\mathrm{H}-1$ to $\mathrm{H}-2$, the chord

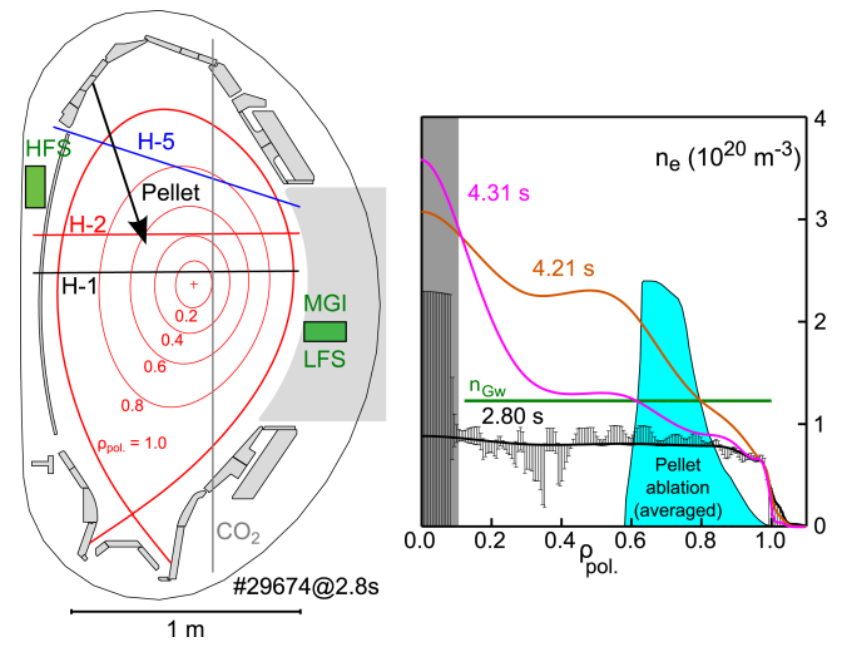

Fig. 1. Left: Equilibrium of discharge \#29674 at 2.8 s (just befor pellet injection) together with the pellet injection trajectory and several interferometer chords for the measurement of the line integrated density. The poloidal position of the two massive gas injection valves (see. Sec. VI) is shown as well. Right: Density profiles just before pellet injection (2.8s), just after the last pellet (4.21s) and $100 \mathrm{~ms}$ later. For details of the pellet ablation please refer to the text.

which is approximately tangential to the flux surfaces near $\rho_{\text {pol }}=0.4$, indicates the inner part of density profile becoming even more peaked at the end of pellet train. This evolution reaches its maximum after about $100 \mathrm{~ms}$ before it rolls over into the normal decay. The left part of Fig. 1 shows the equilibrium of discharge \#29674 together with the pellet injection trajectory, DCN laser interferometer chords $\mathrm{H}-1, \mathrm{H}-2$, $\mathrm{H}-5$ and the $\mathrm{CO}_{2}$ interferometer chord. Density profiles obtained by integrated data analysis for the initial plasma before pellet injection $(2.80 \mathrm{~s})$, immediately after the last pellet $(4.21 \mathrm{~s})$ and at the time when the ratio $\mathrm{H}-1 / \mathrm{H}-5$ reaches its maximum (4.31 s) are shown on the right. Because the lack of density measurements inside $\rho_{\text {pol }} \approx 0.1$ (error bars are displayed for the initial profile only), the region with diverging error for the high density cases is indicated by the shaded area. The pellet ablation radiation, mapped onto the designated pellet trajectory assuming a constant pellet speed of $570 \mathrm{~m} / \mathrm{s}$ and averaged for the concluding 7 pellets of the train, is shown as well as the value of the Greenwald density. The profile evolution is almost adiabatic, indicated by the evolution of the local pressure calculated from Thomson scattering data. The massive pellet driven core density increase, and the simultaneous decrease in temperature, could reduce anomalous transport (i.e. diffusion). At the same time, due to the reduced electron temperature, a larger toroidal electric field develops, boosting the Ware pinch. Modelling is under way in order to test this interpretation. 


\section{B. Pushing the Normalized Divertor Power Flux Towards ITER Values}

Different states of divertor detachment have been investigated in high power AUG discharges by the combination of a high divertor neutral pressure and nitrogen seeding. Fig. 2a shows the operational diagram of core radiation and divertor power flux in ASDEX Upgrade together with the envisaged parameters for ITER and DEMO [6]. The lines represent the possible parameter space for a given $P_{\text {heat }} / R$. A transition to a pronounced detachment state is observed with very moderate target peak power load $\left(<2 \mathrm{MWm}^{-2}\right.$, and a much lower plasma power load as measured by Langmuir probes) even at high values of power fed into the separatrix $\left(\mathrm{P}_{\text {sep }}\right)$. The transition from partial to pronounced detachment appears gradual in target heat flux or pressure. Therefore, the vanishing of the thermoelectric current integrated over the outer target is used as transition marker. Pronounced detachment is accompanied by a substantial rise of pedestal and core densities and reduced ELM amplitudes. For pronounced detachment, a highly radiative region inside the $\mathrm{X}$-point develops. The required enhancement of the neutral pressure and the seeding level leads to performance degradation in AUG, where $\mathrm{H} 98_{\mathrm{y} 2}$ is reduced to values of $0.7-1.1$ for pronounced detachment depending on experimental conditions (see Fig. 2c). More work is required to extrapolate this behaviour to DEMO parameters, but detachment with acceptable confinement may well be possible, with the benefit of a simpler technical divertor solution with reduced heat loads. Operation with cold plasma conditions in front of the target plates may also support the achievement of a small or mitigated ELM regime, and will be mandatory for the achievement of an acceptable target erosion rate. A high neutral divertor pressure is also required in AUG during argon (Ar) or krypton (Kr) seeding used for enhancement of the core radiation. Seeding of $\mathrm{Ar}$ or $\mathrm{Kr}$ at low neutral pressure, hot divertor conditions can lead to a substantial reduction of the type-I ELM frequency, which finally results in core tungsten accumulation. Two thirds of the ITER value of the divertor exhaust parameter $\mathrm{P}_{\text {sep }} / \mathrm{R}$, have been realized in AUG under benign divertor conditions with a divertor neutral pressure of $4 \mathrm{~Pa}$ using the new feature of the switchable He valve of the cryo-pump (the pumping speed was reduced to $1 / 3$ of its design value). Local divertor parameters are expected to approach those in ITER in absolute values under these conditions. AUG has not hit a limit in $\mathrm{P}_{\text {sep }} / \mathrm{R}$ so far, which could, e.g., be caused by a saturation of divertor radiation due to a shrinking of the radiating zone. The use of nitrogen is limited indirectly by MHD effects as $\beta_{\mathrm{N}}$ exceeds the value of 3 in connection with nitrogen induced confinement improvement. Higher values of $\mathrm{P}_{\text {sep }} / \mathrm{R}$ and more pronounced detachment are expected to be achieved with higher divertor neutral pressure. Some operational challenges appeared in AUG so far, which will not apply to larger devices to this extent: The density rise during pronounced detachment (see Fig. 2b) can lead to central plasma densities above the X-2 cutoff value for ECRH, bearing the threat of central tungsten accumulation due to insufficient central heating power density. In ITER and DEMO the cut-off densities will be significantly higher due to the higher magnetic field resulting in higher ECRH frequencies. In AUG, the foreseen new ICRF antennas (see Sec. VII) should enhance the capability for central heating while keeping the tungsten source low. The $\mathrm{T}_{\text {div }}$ sensor, based on a simple passive current measurement in the divertor, proved very useful for the detection of divertor detachment. It is expected to be feasible also for dedicated detachment control, which is foreseen in the near future.
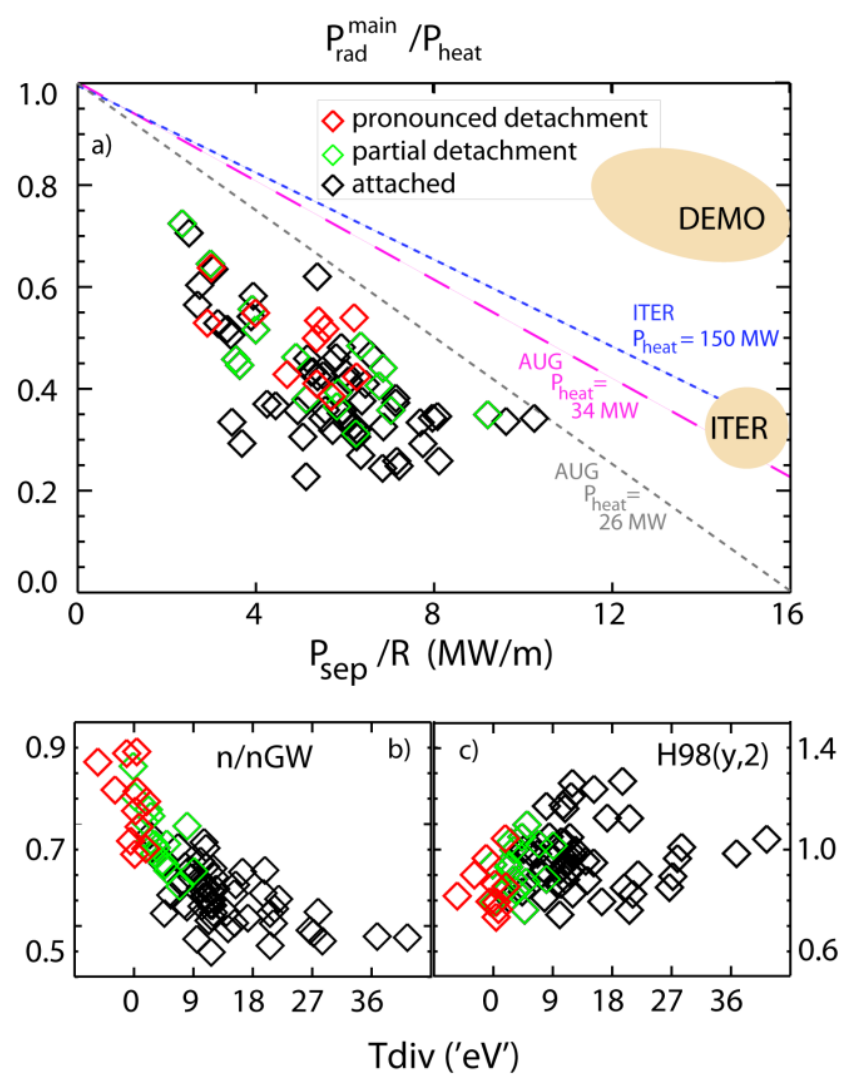

Fig. 2. Operational diagram of core radiation and divertor power flux in ASDEX Upgrade and envisaged parameters for ITER and DEMO [6]. Each point represents a quasi-stationary discharge phase of at least 100 ms regarding gas puff rates and stored energywith the colors denoting different detachment states. The lines represent the possible parameter space for a given $\mathrm{P}_{\text {heat }} / \mathrm{R}$. Main chamber and divertor radiated power are taken from real time evaluation. (b) Greenwald fraction versus $\mathrm{T}_{\text {div. }}$ (c) $\mathrm{H} 98_{\mathrm{y} 2}$ versus $\mathrm{T}_{\text {div }}$ (please note: the divertor temperaure is evaluated from thermo-electric currents which means that in the case of pronounced detachment they lose their physical validity and ' $\mathrm{T}_{\text {div }}$ ' $<0$ can appear).

\section{SCENARIO DEVELOPMENT FOR ITER}

ITER baseline demonstration discharges were performed in AUG at $\mathrm{I}_{\mathrm{P}}=1.1$ and $1.2 \mathrm{MA} \mathrm{q}_{95}=3$ with central ECRH or ICRH, respectively [7]. Values for density and energy confinement were kept simultaneously close to the requirements of the ITER baseline scenario and a stable behavior for many confinement times could be achieved as long as $\beta_{\mathrm{N}}$ stayed above 2 (typically $2.0<\beta_{\mathrm{N}}<2.2$ ). At lower heating power and thus at $\beta_{\mathrm{N}}=1.8$ so far only $\mathrm{H}$-factors around 0.85 have been achieved. Such low heated discharges were only stationary at $\mathrm{D}_{2}$ puffing rates around $3 \cdot 10^{22} \mathrm{~s}^{-1}$ and with a freshly boronized 
wall, otherwise, accumulation of tungsten in the plasma center occurred.

As a possible alternative, the $\mathrm{q}_{95}=3.6$ scenario has been investigated. The operational window allowing stationary behavior at the higher safety factor is significantly larger compared to the $\mathrm{q}_{95}=3$ scenario.

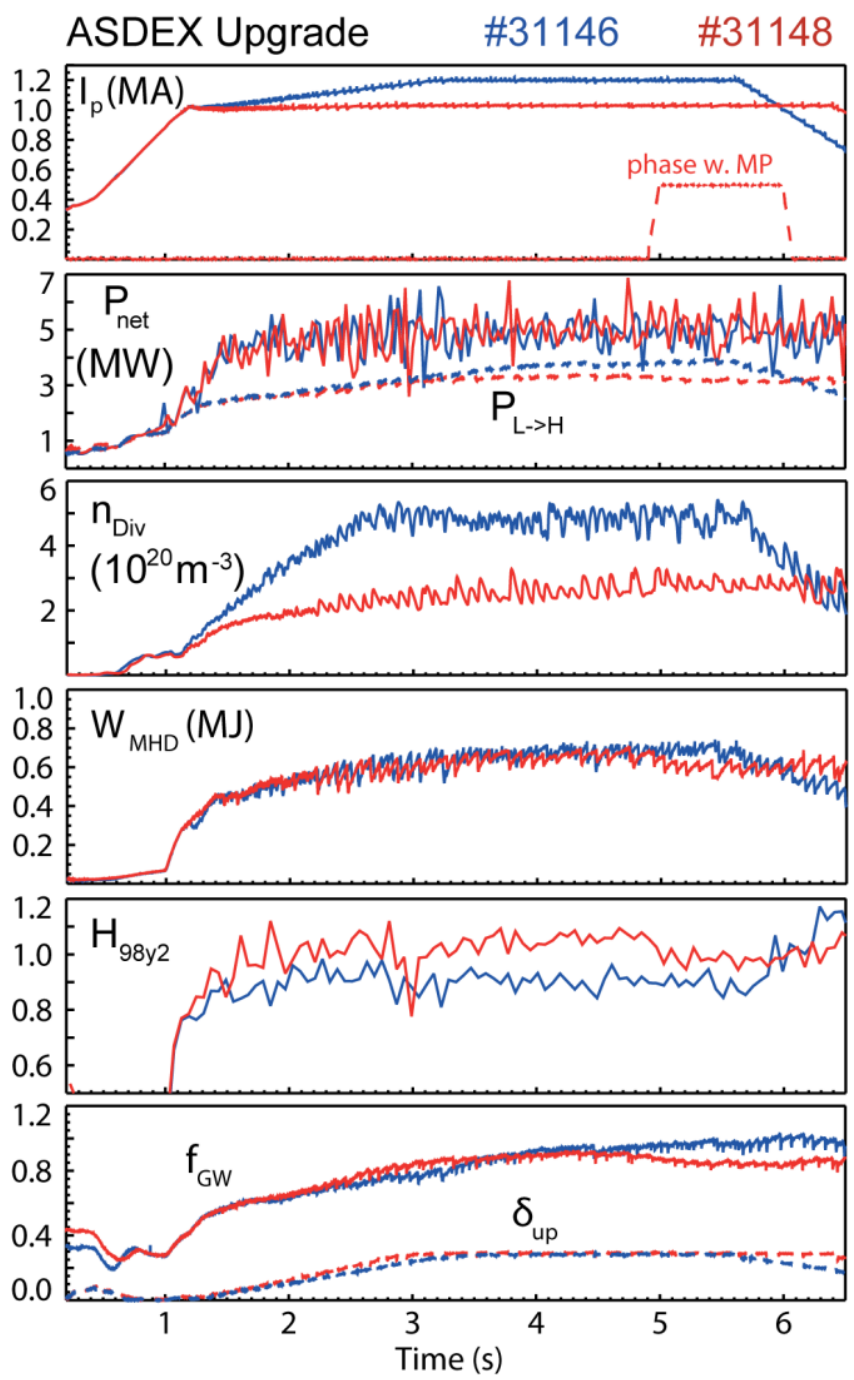

Fig. 3. Time traces of various parameters (plasma current $I_{p}$, net heating power $\mathrm{P}_{\text {net }}, \mathrm{H}$-mode power threshold $\mathrm{P}_{\mathrm{L}>\mathrm{H}}$, neutral gas density in the divertor $\mathrm{n}_{\mathrm{DIV}}$, stored energy $\mathrm{W}_{\mathrm{MHD}}, \mathrm{H}$-mode factor $\mathrm{H} 98_{\mathrm{y} 2}$, Greenwald fraction $\mathrm{f}_{\mathrm{GW}}$, upper triangularity $\delta_{\mathrm{up}}$ ) for a comparison of a $\mathrm{q}_{95}=3$ (\#31146, blue) with a $q_{95}=3.6$ (\#31148, red) discharge. For both discharges the toroidal field $\mathrm{B}_{\mathrm{t}}=2 \mathrm{~T}$, the applied heating power (NBI+ ICRH) as well as the plasma shape are the same.

Lower $\mathrm{D}_{2}$ puff rates are possible even under conditions of a diminishing boronization. Discharges with high normalized density and at the target $\beta_{\mathrm{N}}$ value of 2.2 have been established. Fig. 3 shows the comparison of a $\mathrm{q}_{95}=3$ (\#31146, blue) and a $\mathrm{q}_{95}=3.6$ (\#31148, red) which were run in the same way except for the reduction of the plasma current from 1.2 MA to 1.0 MA and a reduced gas puff $\left(1.5 \cdot 10^{22} \mathrm{~s}^{-1}\right.$ instead of $\left.3 \cdot 10^{22} \mathrm{~s}^{-1}\right)$ in the latter case. As can be judged from Fig.3 discharge \#31148 is stable even at this lower gas puff rate. Due to the higher $\mathrm{H}$ factor (1.05 instead of 0.91$)$ a very similar performance could be achieved at the lower current. However, to satisfy the ITER requirement $\mathrm{H} 98_{\mathrm{y} 2}=1.2$ would be necessary at the higher $\mathrm{q}_{95}$. In \#31148 a $\mathrm{n}=2$ magnetic perturbation (MP field was switched on between $4.8 \mathrm{~s}$ and $6 \mathrm{~s}$ ) in order to test its influence on ELMs. Unfortunately the application of MP leads only to a degradation of both the energy and the particle confinement, but has no effect on ELMs.

Generally, very large ELMs occurred in both scenarios, which appear difficult to mitigate. Three mitigation techniques (pellets, MP (see also Sec. IV) and N-seeding) have been tried, but did not show the desired effect. The solution of this problem remains the biggest challenge for optimizing such plasmas towards divertor heat load mitigation under steadystate conditions. However, ITER will operate at a much lower collisionality because of the much higher pedestal temperature and therefore mitigation as described in Sec. IV could be a solution. As a side-result type-II ELM phases [8] could be triggered by shifting the plasma closer to a double-null configuration. Seeding of nitrogen shows the first promising results in terms of improved confinement and reduced divertor temperature, but in both scenarios long-lasting stationary behaviour with $\mathrm{N}$ seeding could not be reached until now. Finally for the operation in helium, which is foreseen in the early operational phases of ITER, no major difficulties were observed [7].

\section{ELM Mitigation With Magnetic PERTURBations AT LOW COLLISIONALITY}

Motivated by the requirement of mitigating the fast heat loss caused by ELMs in ITER and the success of suppressing large, type I ELMs in DIII-D at low pedestal collisionalities $\nu *_{\text {ped }}$ [9], ASDEX Upgrade had been equipped with a set of 16 in-vessel saddle coils at the low-field side, mounted in two rows of eight coils, one row located above and one below the mid-plane [10], to produce non-axisymmetric magnetic perturbations (MP) with toroidal mode numbers up to $n=4$. With this new system, a high density regime of ELM mitigation has been identified earlier [11].

Recent efforts in AUG were concentrated on the ELM suppression regime for low $v^{*}$ ped [12]. The cases investigated are characterized by $\mathrm{T}_{\text {ped }} \geq 1.0 \mathrm{keV}, \mathrm{n}_{\text {ped }}=3.2 \cdot 10^{19} \mathrm{~m}^{-3}, \mathrm{v}_{\text {ped }}^{*} \leq 0.3$ (for typical $\mathrm{Z}_{\mathrm{eff}}=1.4$ ). Discharges with slightly increased gas puffing or He injection, with $\mathrm{T}_{\text {ped }}=0.75 \mathrm{keV}, v_{\text {ped }}^{*}=0.44$, show almost no effect of MPs on type I ELMs and density, hence we conclude that there is a threshold in between these values. With the available coils toroidal mode numbers $n=1,2$ and 4 can be achieved, depending on the wiring of the coils. The poloidal mode number spectrum is always broad, due to the box shaped MP in two poloidally relatively narrow regions. However the field component with poloidal mode number $\mathrm{m}=$ $\mathrm{n} \cdot \mathrm{q}$, resonant with the plasma safety factor $\mathrm{q}$ on a surface, can be efficiently varied by varying the phase difference $(\Delta \Phi)$ between the field patterns in the upper and lower coils. This technique, applicable with $n=1$ and $n=2$, allows to scan the 
MP alignment with respect to the plasma field without ramping the plasma safety factor $\mathrm{q}$ and therefore without changing other plasma parameters. The reaction of type I ELMs and plasma density to magnetic perturbations depends critically on the structure of the applied perturbation field. Fig. 4 shows time traces of a discharge for which the differential phase angle between the $n=2$ current pattern in the upper and lower coil rings is continuously varied. Classical nonstationary ELM-free $\mathrm{H}$-mode with accumulation of plasma density and impurity radiation are triggered at $\mathrm{t}=2.85 \mathrm{~s}$. Once triggered, their intrinsic transport dynamics dominates the effect of the

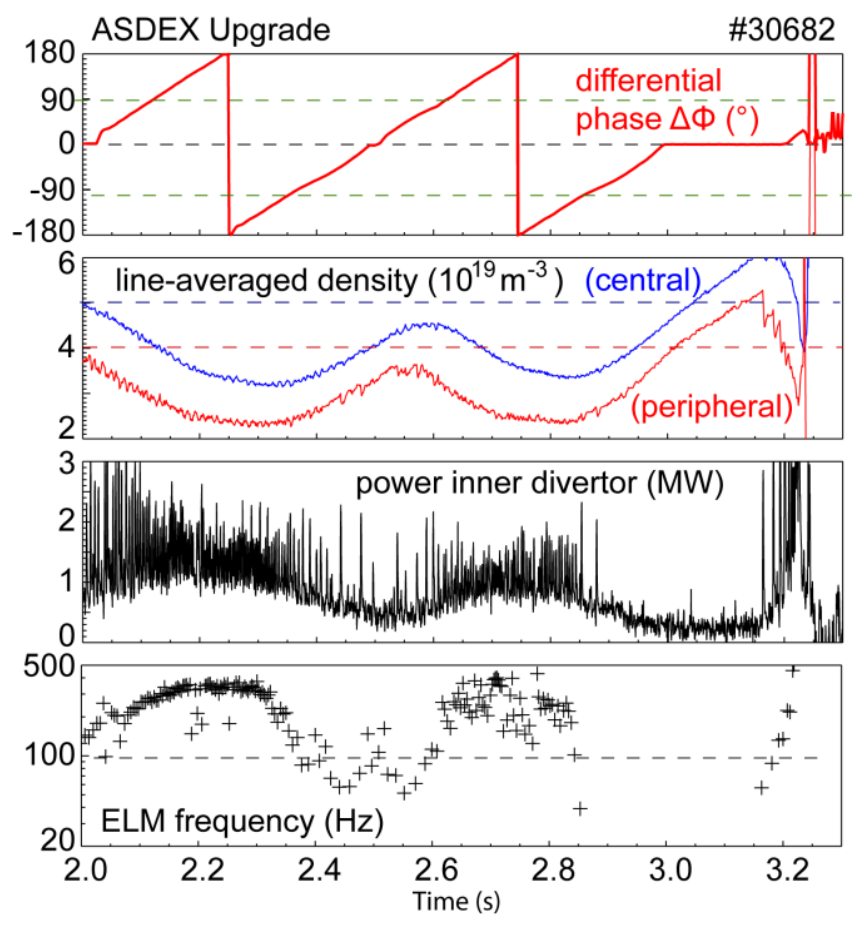

Fig. 4. Time traces of \#30682 with $\mathrm{n}=2$ magnetic perturbation and continuously varied differential phase $\Delta \Phi$ between upper and lower coil rings.

$\Delta \Phi$ ramp. Therefore, the $\Delta \Phi$ ramp is conducted in both directions (not shown here).

A window for triggering ELM-free H-mode by MPs, determined from the respective onset condition, is between $\Delta \Phi \sim-90^{\circ} \ldots-40^{\circ}$. In the ELM-free phase, $\Delta \Phi=0^{\circ}$ is held from $\mathrm{t}=2.9 \mathrm{~s}$ to $3.2 \mathrm{~s}$, resulting in its sustainment until it is terminated by a big ELM. For practically all other values of $\Delta \Phi$, the density decreases and the ELM frequency increases over their values without MP (dashed lines in Fig.4) to some extent. The lowest plasma density along with strongest ELM mitigation, is obtained near $\Delta \Phi=-150^{\circ}$ and $\Delta \Phi=+52^{\circ}$ (from a reversed scan in \#30826). In a discharge with low $v^{*}$ ped and optimum setting of $\Delta \Phi(\# 31128)$ a clear mitigation of type I ELMs for the whole period of the applied MP (3s) is observed [12]. The peak target power is reduced from $8-11 \mathrm{MW}$ to $\leq 1$ MW during ELM peaks in the inner divertor and from 6-10 $\mathrm{MW}$ to $\leq 2 \mathrm{MW}$ in the outer divertor. The inter-ELM power, especially in the inner divertor, increases slightly. Accompanied with ELM mitigation there is a strong electron density reduction, both at the edge and in the core. The central line averaged density drops from $\mathrm{n}_{\mathrm{e}}=4 \cdot 10^{19} \mathrm{~m}^{-3}$ to $\mathrm{n}_{\mathrm{e}}=2.6 \cdot 10^{19}$ $\mathrm{m}^{-3}$, i.e. by $35 \%$ of the original value. Similarly, the MHD stored energy decreases from $\mathrm{W}_{\mathrm{MHD}}=590 \mathrm{~kJ}$ (pre-ELM, MP off) to $360 \mathrm{~kJ}$ with MP, i.e. by a similar factor. The ion temperature on top of the pedestal, at poloidal flux radius $\rho_{\text {pol }}=0.87$ drops from $T_{i}=1.8$ to $1.3 \mathrm{keV}$, whereas the electron temperature $\left(\mathrm{T}_{\mathrm{e}}=1.0-1.2 \mathrm{keV}\right)$ changes little. There is a noticeable braking of plasma rotation in the core up towards the channel at $\rho_{\mathrm{pol}}=0.87$, and a smaller change of rotation at $\rho_{\text {pol }}=0.93$, i.e. the pedestal rotation profile becomes flatter with MP on.

First MHD plasma response calculations using MARS-F show that the resonant (field-aligned) field component is predicted to be always shielded very strongly [13]. Therefore, strongest plasma response is expected to be shifted somewhat off pitch alignment of the externally applied field, where the plasma response can amplify the perturbation. The strongest pitch-aligned resonant field amplitude is predicted to be shifted significantly from its vacuum location, possibly driven by resonantly amplified low $\mathrm{n}$ edge localized peeling modes. This prediction agrees with the experimental observation of most efficient ELM mitigation.

\section{ELECTRON CYCLOTRON CURRENT DRIVE EXPERIMENTS}

\section{A. NTM Suppression by Feedback Controlled ECCD}

The NTM control experiments at ASDEX Upgrade are based upon a complex interplay between multiple real-time diagnostic systems, the central discharge control system (DCS) and its capability of directly controlling the actuator ECCD, both in power (typically on/off) and deposition (by tilting launch mirrors) [14]. Lowest latencies are required in each subsystem to allow the overall control loop to reach timescales faster than the typical energy confinement time (here: $\tau_{\mathrm{E}} \approx 60 \mathrm{~ms}$ ). The most relevant involved diagnostics for the control are the real-time equilibrium, the ECE measurements, the electron density and Mirnov coils and a real-time version of the TORBEAM code. The latter is a highly accelerated version of the beam-tracing code TORBEAM [15] complemented by an interface to the DCS system. It receives in real-time the equilibrium, density profile and further plasma parameters $\left(\mathrm{I}_{\mathrm{P}}, \beta_{\mathrm{pol}}\right)$ to calculate by numerical integration the propagation path of the ECCD beam. It provides coordinates $\mathrm{R}, \mathrm{z}, \Phi$ and $\rho_{\text {pol }}$ of the peak power deposition. This is close enough to the peak of driven current density and is used as a proxy for this quantity.

In order to provide reliable conditions for the production of high beta NTMs experiments $\mathrm{H}$-mode discharges with specific boundary conditions are typically used as a target. To enhance confinement and at the same time protect the divertor, nitrogen seeding (see also Sec. IIB) is applied. The toroidal field is set to $2.5 \mathrm{~T}$ on axis to allow $140 \mathrm{GHz} \mathrm{ECRH}$ to be used centrally for impurity control. This leaves up to three 
gyrotrons for ECCD at the NTM location, taking place above and below the plasma center. High beta operation $\left(\mathrm{P}_{\text {aux }}>10\right.$ $\mathrm{MW}$ ) ensures that the NTM, once triggered, grows to its saturated size. In order to ensure the triggering of the instability, a power ramp using neutral beam injection (NBI) of up to $17.5 \mathrm{MW}$ is used (see Fig. 5). Once the mode is detected, the DCS enters the control mode meant to stabilize the NTM.

With accurate and fast deposition control together with the intelligent features of DCS, a new stabilization technique previously used at TCV [16] has been implemented at

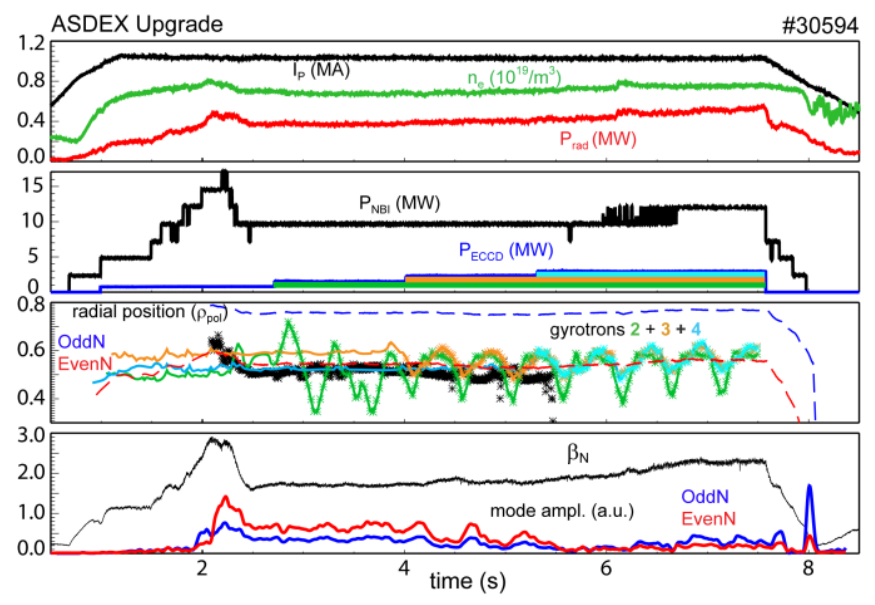

ASDEX Upgrade. Instead of trying to determine the absolute

Fig. 5. Time traces of \#30594 with automatic NTM stabilization using 3 gyrotrons. Whereas the position of the first gyrotron is fixed at the plasma center, the three successive ones (from $t=2.7 \mathrm{~s}$ on) are steered accross the measured / preempted position of the 3/2 NTM. The signals from top to bottom are: plasma current $I_{p}$, central line averaged $n_{e}$, total radiated power $\mathrm{P}_{\text {rad }}$ (top inset); NBI power $\mathrm{P}_{\mathrm{NBI}}$ and ECCD power $\mathrm{P}_{\mathrm{ECCD}}$ ( $2^{\text {nd }}$ inset); radial positions (in terms of $\rho_{\text {pol }}$ ) of the $\mathrm{q}=1.5$ and $\mathrm{q}=2$ surfaces from the realtime equilibrium EvenN and OddN, of the $3 / 2$ mode from realtime ECE reconstruction and of the three deposition of the gyrotrons used for ECCD ( $3^{\text {rd }}$ inset); normalized plasma pressure $\beta_{\mathrm{N}}$ and amplitudes of the MHD modes with EvenN and OddN (bottom inset).

best deposition location using localization techniques or equilibrium reconstructions with internal constraints, one can accept offset errors and counteract them by sweeping around the estimated position. In this way, it is guaranteed that the ideal deposition location is at least met periodically. As soon as enough current is driven in this position, the NTM is rapidly stabilized. Discharge \#30594 (see Fig. 5) has been set up to test the necessary power for stabilization of a $3 / 2$ NTM at $\beta_{\mathrm{N}} \approx 2$ using the sweeping method. When the stabilization begins $(t=2.2 \mathrm{~s})$, the gyrotron launchers move into position. The ECCD power is continuously increased until at about $\mathrm{t}=4.3 \mathrm{~s} 2 \mathrm{MW}$ of ECCD are launched, driving in total a current of about $30 \mathrm{kA}$. While the power of one gyrotron only weakly modulates the NTM amplitude signal (10-20\%), 1.5 MW lead to a significant $(50 \%)$ modulation in the amplitude, always correlated with the ECCD deposition crossing the NTM location. Only the addition of the third current-driving EC beam finally pushes the instability clearly below its marginal size, immediately on the first pass through the NTM location, indicating a very fast response to the locally driven current. The stabilization of the mode consecutively leads to a rising $\beta_{\mathrm{N}}$. Subsequent sweeping near the $3 / 2$ rational surface does not retrigger the instability; it rather keeps preemptively stabilizing EC power near the rational surface. With the stabilization of the NTM, the DCS starts a slow NBI power ramp which is able to raise beta to above 2.2 without reappearance of the NTM.

Without any prior conditioning or pre-selection, the system can target either $2 / 1$ or $3 / 2$ NTMs automatically by dynamically setting the proper launching parameters This works reliable even within the same discharge as it is shown in [14].

\section{B. Steady State Current Profile Tailoring}

Experiments with central ctr-ECCD have been performed in order to modify the q-profile for accessing advanced scenarios. This procedure has been chosen deliberately (instead of doing co-ECCD off-axis) in order to make use of the higher current drive efficiency at the elevated temperatures in the center. According to TORBEAM calculations a centrally peaked ctr-ECCD profile could be achieved with a maximum value of $70 \mathrm{Mam}^{-2}$. As a result, a strong reversal of the q-profile with a minimum $\mathrm{q}$ slightly above 1 and $\mathrm{q}_{0} \geq 4$ could be stationary achieved. In parallel, an internal electron transport barrier has been be triggered in the region of the inversed q-profile. Further studies aim at optimizing the ECCD deposition profile in order to achieve improved $\mathrm{H}$ mode scenarios, showing an increase in confinement at still good MHD stability with flat elevated q-profiles at values between 1.5 and 2 .

\section{PRODUCTION AND Mitigation OF RUNAWAY ELECTRONS BY MASSIVE GAS INJECTION}

Runaway electrons (REs) are expected to be generated in ITER disruptions at any significant plasma current. While the generation mechanisms of REs are believed to be understood, there is no consent on which loss mechanisms are dominant in present experiments, and which one will prevail in ITER.

ASDEX Upgrade disruptions - independently of mitigation or plasma parameters - do not exhibit formation of $\mathrm{RE}$ beams. A scenario for RE generation during the thermal quench was established during the 2014 campaign by injecting up to 0.6 bar.l of argon in a low density circular plasma, with a current of 0.8 MA and $2 \mathrm{MW}$ of ECRH heating [17]. The injected argon induces a fast quench of the current carried by thermal electrons, followed by a long-lived runaway beam, carrying a current of up to $300 \mathrm{kA}$, and lasting up to $320 \mathrm{~ms}$ (see Fig. 6). The circular plasma is vertically stable; the position, the form of the plasma and the current profile from equilibrium reconstruction are in agreement with soft X-Ray profiles.

In some of the RE beams generated, argon was injected a second time (with a second valve) with a delay of $70 \mathrm{~ms}$ after the first argon puff. The RE current decay rates, after both the first and the second argon puff, show a clear dependence on 
the argon amount injected - i.e. the more gas the faster the decay (see Fig. 6) - suggesting that electron energy loss by collision (friction and radiation induced) with the high $\mathrm{Z}$ impurity atoms is a significant RE dissipation mechanism. However, although the effect is rather obvious from the experimental data a detailed explanation of the processes at work is still missing since the deduced density of Ar ions is too small to explain quantitatively the quenching of the REs [17]. Simulations of the generation and decay of the RE beam are currently underway taking into account the available fast line integrated density measurements and the $\gamma$ spectra from a $\gamma /$ neutron spectrometer.

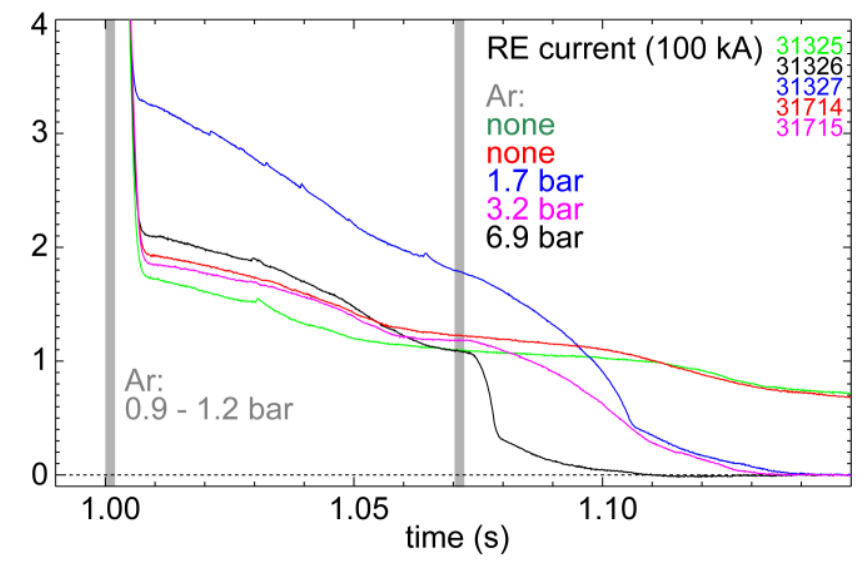

Fig. 6. Plasma current evolution in circular plasmas with an initial current of $800 \mathrm{kA}$ after two consecutive massive gas injections (except \#31325 and \#31714 with only one MGI). The values given in bar denote the filling pressure of the MGI valve.

\section{HARDWARE UPGRADES IN SUPPORT OF INVESTIGATIONS FOR ITER \& DEMO}

During the last shut down, which was concluded in May 2015, two 3-strap ICRH antennas were installed in ASDEX Upgrade (see Fig. 7) in order to reduce $W$ release due to sputtering from sheath accelerated plasma ions [18,19]. The idea behind the antenna design was to reduce the image currents at the antenna frame and thus the RF electric fields which drive rectified sheaths and $\mathrm{W}$ sputtering. The antennas are equipped with 3 (chosen out of 10) locations for reflectometry measurements, 11 lines of sight visible spectroscopy and local RF current measurements imbedded in the antenna frame. This sophisticated diagnostics together with the gas outlets integrated in one of the neighboring old antennas allow optimizing the coupling of the ICRF power into the plasma. First results achieved during the initial operation of the 2015 campaign point indeed to a noticeable reduction of the $\mathrm{W}$ source at the cost of a reduced coupling which was expected because of the higher $\mathrm{k} \|$. The local $\mathrm{W}$ source which was spectroscopically measured at the antenna frame correlates strongly with the measured local RF currents.
The results will be described in details in a separate publication. Over the next two years the old ECRH system with four $0.5 \mathrm{MW} / 2 \mathrm{~s}$ gyrotrons will be exchanged to 4 new ones with $1 \mathrm{MW} / 10 \mathrm{~s}$ each. This will increase the total EC power to 7-8 MW in total, allowing a versatile tailoring of the deposition profile and a mixture of heating and current drive settings being well above the L-threshold with EC heating alone. In order to make full use of the available $34 \mathrm{MW}$ of auxiliary heating power the power supplies will be strengthened accordingly. In the domain of plasma wall interaction the characteristics of an all metal wall will be exploited further. Specifically the new divertor manipulator will be used to perform experiments with differently shaped divertor tiles as well as to test actively cooled divertor PFCs. The $\mathrm{W}$ coated tiles at the central column will be exchanged to massive steel tiles to explore their capabilities as PFCs in low power load areas as well as the influence of their ferromagnetism on plasma operation. The tiles will be produced from Eurofer and from P92, which has similar properties as Eurofer (except for its activation) but is more readily available. On top of these hardware changes the available diagnostic will be updated and improved. On the near term list are the development and installation of an imaging MSE system, to improve the capabilities for the measurement of the current profile evolution in advanced scenarios and a divertor Thomson scattering system, to supply spatially resolved measurements of the electron density and temperature. The latter are of specific importance as input for simulations to understand and extrapolate the physics of exhaust scenarios.

\section{SUMMARY AND CONCLUSION}

Recent experiments in ASDEX Upgrade aimed at improving the physics base for ITER and DEMO to prepare operation and aid the design. In order to increase its exhaust capabilities and operational flexibility a new bulk W divertor as well as an adjustable cryo-pump had been installed prior to the 2014 campaign. Due to its large installed heating power a large normalized heat flux $\mathrm{P}_{\text {sep }} / \mathrm{R}=10 \mathrm{MWm}^{-1}$ has been reached, representing $2 / 3$ of the ITER value, under partially detached conditions with a peak target heat flux well below $10 \mathrm{MWm}^{-2}$. The divertor load could be further reduced by increasing the core radiation while still keeping the confinement in the range of $\mathrm{H} 98_{\mathrm{y} 2} \approx 1$. Future studies at even higher auxiliary heating power will allow almost matching the ITER value and - if successful - may even hint to a possible divertor solution for DEMO. In previous experiments with magnetic perturbation (MP) coils ELM suppression has been achieved at high collisionality in a wide window of $\mathrm{q}_{95}$ and perturbation spectrum, with no clear indication of a resonant process. Due to the increased pumping speed which became possible through a redesign of the outer divertor and the full availability of the four $1 \mathrm{MW}$ gyrotrons, ELM suppression experiments at low collisionality could be performed. Differently to the observation at high $v^{*}{ }_{\text {ped }}$, ELM mitigation was only observed in a narrow spectral window. According to code simulations, this hints to resonantly amplified low-n edge localised peeling modes. Together with the results achieved in other Tokamaks theses observation may provide deeper insight 
and ultimately to a predictive capability for the ELM mitigation via MP. The ITER $\mathrm{Q}=10$ baseline scenario has been investigated, matching as close as possible the triangularity, the plasma beta, $\mathrm{q}_{95}$ and the distance the L-H threshold. It turned out that the ELM frequency is low and consequently the energy ejected by a single ELM is very high. At the same time ELM mitigation seems to be difficult. At the moment it is not clear, whether in ITER a similar behaviour will be observed. By matching the above mentioned parameters the edge plasma of the AUG discharges is in a very different collisionality regime compared to the one of ITER, which could have strong implications for the (neoclassical) transport. Irrespectively, an H-Mode scenario has been developed as a possible alternative achieving a similar performance at lower plasma current (and consequently higher $\mathrm{q}_{95}$ ). In disruption mitigation studies with massive gas injection (MGI) a runaway electron beam could be provoked and mitigated by a second MGI. Although the experimental evidence is very clear, a detailed explanation of the processes at work is still missing. Experiments using ECCD

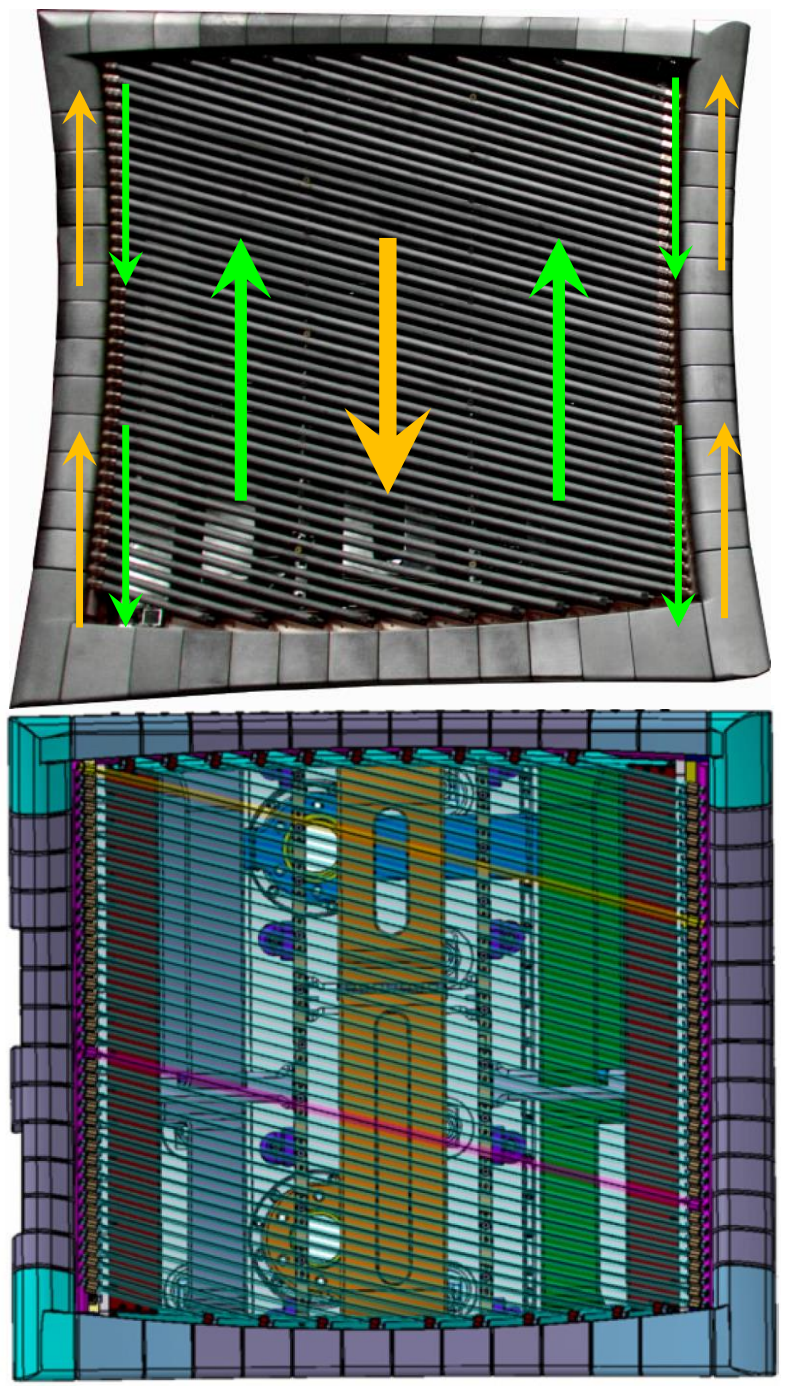

Fig. 7. The three-strap antenna as mounted in AUG (top). The colored arrows indicate the image currents which can be reduced by tayloring the voltage to the antenna straps. The CAD drawing (bottom) of the antenna shows the arrangement of the straps behind the Faraday screen. with feedback controlled deposition have allowed successfully testing several control strategies for ITER, including automated control of $(3,2)$ and $(2,1)$ NTMs during a single discharge. In the stabilized discharges the confinement can be recovered leading to a normalized beta above 2 and the suppressing the reappearance of NTMs. Concerning advanced scenarios, experiments with central ctr-ECCD have been performed in order to modify the q-profile. This procedure has been chosen deliberately (instead of doing co-ECCD off-axis) in order to make use of the higher current drive efficiency. A strong reversal of the q-profile could be stationary achieved and an internal transport barrier could be triggered. Ongoing and planned enhancements aim at further strengthening of the heating systems and the corresponding power supplies. Together with the foreseen upgrades of the diagnostic systems, ASDEX Upgrade aims at playing an important role in the future investigations aimed at the closing of gaps for the operation of ITER and the design of DEMO.

\section{ACKNOWLEDGMENT}

The authors would like to thank the ASDEX Upgrade team and the European MST1 team for their continuous and strong support. This work has been carried out within the framework of the EUROfusion Consortium and has received funding from the Euratom research and training programme 2014-2018 under grant agreement No 633053. The views and opinions expressed herein do not necessarily reflect those of the European Commission.

\section{REFERENCES}

[1] H. Zohm et al., , Nucl. Fusion 55 (2015) 104010

[2] F. Romanelli et al., "Fusion Electricity - A roadmap to the realisation of fusion energy", European Fusion Development Agreement (EFDA) 2012, ISBN 978-3-00-040720-8

[3] A. Herrmann et al., Nucl. Fusion 55 (2015) 063015

[4] T. Eich et al., Nucl. Fusion 53 (2013) 093031

[5] P. Lang et al., "Controlled fuelling of high density scenarios at ASDEX Upgrade in support of ITER and DEMO", P1.127, 42nd EPS Conference on Plasma Physics, Lisbon, June 22-26, 2015

[6] A. Kallenbach et al., Nucl. Fusion 55 (2015) 053026

[7] J. Schweinzer et al., "Development of the Q=10 Scenario for ITER on ASDEX Upgrade (AUG)", Proc. of the $25^{\text {th }}$ IAEA Fusion Energy Conference, St. Petersburg, RF (2014), EX/9-4, IAEA Vienna

[8] E. Wolfrum et al., Plasma Physics and Controlled Fusion 53, 8 (2011), 085026

[9] T.E. Evans et al., Nucl. Fusion 48 (2008) 024002

[10] W. Suttrop et al., Fusion Engineering and Design 84 (2009) 290

[11] W. Suttrop et al., Plasma Physics and Controlled Fusion 53 (2011) 124014

[12] W. Suttrop et al., "Studies of Magnetic Perturbations in HighConfinement Mode Plasmas in ASDEX Upgrade", Proc. of the 25th IAEA Fusion Energy Conference, St. Petersburg, RF (2014), EX/P1-23, IAEA Vienna

[13] D.A. Ryan et al., "Simulations of a resonant plasma kink response to externally applied magnetic perturbations in the AUG tokamak", Joint EU-US Transport Task Force Workshop (2014) Culham, U.K

[14] M. Reich et al., "Real-time Control of NTMs Using ECCD at ASDEX Upgrade", Proc. of the 25th IAEA Fusion Energy Conference, St. Petersburg, RF (2014), PPC/P1-26, IAEA Vienna

[15] E. Poli et al., Comp. Phys. Com. 136 (2001), 90 
[16] D. Kim et al, "Stabilization of NTMs using real-time equilibrium reconstruction on TCV", 55th APS conference on Plasma Physics, Bull. Am. Phys. Soc., Volume 58 (2013) PP8.00073

[17] G. Pautasso et al., "Generation and suppression of runaway electrons in ASDEX Upgrade disruptions", P1.134 42nd EPS Conference on Plasma Physics, Lisbon, June 22-26, 2015
[18] V. Bobkov et al., Nuclear Fusion 53 (2013) 093018

[19] V. Bobkov et al., "Progress in controlling ICRF-edge interactions in ASDEX Upgrade", 21st Topical Conference on Radiofrequency Power in Plasmas, Lake Arrowhead, USA (2015) 